\title{
Multisystem inflammatory syndrome in children and Kawasaki disease in infants: 2 sides of the same coin?
}

\section{To the editor,}

The world has been plagued by the novel severe acute respiratory syndrome coronavirus 2 (SARS-CoV-2) causing the global pandemic of coronavirus disease 2019 (COVID-19). A new disease entity, called multisystem inflammatory syndrome in children (MIS-C), emerged in late April to early May 2020, affected clusters of children in Europe and North America, and showed a temporal association with SARS-CoV-2 infection. This article presents an infant with MIS-C and features of Kawasaki disease shock syndrome (KDSS) who was treated in a tertiary hospital in Sabah, Malaysia. This study aimed to assess the laboratory and clinical characteristics of MIS-C in infants, along with its similarities and differences with Kawasaki disease (KD).

A healthy 4-month-old girl was diagnosed with SARS-CoV-2 infection. One month after being diagnosed, she presented with fever, vomiting, rash, bilateral nonpurulent conjunctivitis, dry fissured red lips, and poor feeding. She went into shock 12 hours after admission and was transferred to the pediatric intensive care unit for invasive mechanical ventilation.

Her white blood cell count was $5.8 \times 10^{3} / \mu \mathrm{L}$ (absolute lympho. cyte count, $1.5 \times 10^{3} / \mu \mathrm{L}$ ), her platelet count was $65 \times 10^{3} / \mu \mathrm{L}$, and she had hypoalbuminemia $(24 \mathrm{~g} / \mathrm{L})$. Her inflammatory markers were elevated: C-reactive protein, $102.6 \mathrm{mg} / \mathrm{L}$; and ferritin, 504 $\mathrm{ng} / \mathrm{mL}$. She had a deranged coagulation profile (prothrombin time, 17 seconds; activated partial thromboplastin time, 75.1 seconds; international normalized ratio, 1.45) and an elevated D-dimer level $(2.3 \mu \mathrm{g} / \mathrm{mL})$. Her chest radiography findings were normal.

She had an unremitting fever of up to $39.7^{\circ} \mathrm{C}$ daily. She was hypotensive and required intravenous (IV) adrenaline and noradrenaline. Given this constellation of findings, she received IV immunoglobulin (IVIG) $1 \mathrm{~g} / \mathrm{kg} /$ dose for 2 days, IV methylprednisolone $2 \mathrm{mg} / \mathrm{kg}$ daily for 5 days, and oral prednisolone. She was also empirically treated with IV piperacillin-tazobactam, which was discontinued after the blood culture result was negative. No virus was detected in the respiratory multiplex panel. Her serum SARS-CoV-2 antibody test results were positive for Ig G.

On day 4 of hospitalization, her fever subsided after completion of IVIG therapy. Echocardiography revealed normal coronary arteries and a minimal pericardial effusion. She was weaned off of the vasopressors and extubated on day 7 of hospitalization.
Oral aspirin and subcutaneous enoxaparin were administered to treat thrombocytosis. The patient was discharged 19 days after hospitalization. Follow-up echocardiography 1 month later revealed normal coronary arteries and resolved pericardial effusion.

We compared 5 infants from published case reports in PubMed, who fulfilled case definitions for MIS-C of the Centers for Disease Control ${ }^{1)}$ or World Health Organization ${ }^{2)}$ with our case described above. ${ }^{3-7)}$ These cases were further analyzed to determine if they fulfilled the diagnostic criteria for $\mathrm{KD}$, incomplete $\mathrm{KD}$, or KDSS (Table 1).

None of the infants had any underlying comorbidity except for infant 2, who was born prematurely (gestational age, 26 weeks); had chronic lung disease, swallowing difficulty, and periventricular hemorrhage; and had recently undergone a surgical gastrostomy procedure. ${ }^{3)}$ All infants presented with persistent fever for at least 3-day duration. Among the infants that fulfilled the diagnostic criteria of $\mathrm{KD}$ or incomplete $\mathrm{KD}$, the most consistent features were rash and conjunctival injection. All but infant 2 received IVIG; this was the only infant who died.

MIS-C is a new disease entity with a heterogeneous clinical presentation. It seems to predominantly affect older children, whereby the median age reported was $7-9$ years. ${ }^{8,9}$ The majority of the above infants $(5$ of $6,83 \%$ ) presented with features consistent with $\mathrm{KD}$, incomplete $\mathrm{KD}$, or KDSS with prominent mucocutaneous features (rashes, cracked lips, and conjunctival injection) and cardiovascular involvement. These 5 infants were aged between 2 and 6 months in contrast to the mean age at presentation of typical KD of 3.4 years. ${ }^{10)}$ It is important to note that typical $\mathrm{KD}$ is rarely reported in infants younger than 6 months of age. Furthermore, most MIS-C series reported male-to-female ratios of approximately $1.5: 1$, but there was a female preponderance of $2: 1$ in this cohort.

Multisystemic involvement, especially of the gastrointestinal and cardiovascular systems with shock, was more prominent in MIS-C than in typical KD. Inflammatory markers were markedly elevated in contrast to typical KD. ${ }^{8)}$ Moreover, MIS-C shows many similarities to KDSS, a rare form of KD.

An important prerequisite for the diagnosis of MIS-C is exposure to SARS-CoV-2, confirmed by a positive reverse transcription-polymerase chain reaction (RT-PCR), antigen, or serology test. Past or previous infections were identified in most MIS-C

Corresponding author: Hing Cheong Kok, MD, Sabah Women and Children's Hospital, Karung Berkunci No. 187, 88996 Kota Kinabalu, Sabah, Malaysia 凶Email: jeremykokhc@gmail.com, https://orcid.org/0000-0002-9781-5905

Received: 9 July, 2021, Revised: 21 August, 2021, Accepted: 23 August, 2021

This is an open-access article distributed under the terms of the Creative Commons Attribution Non-Commercial License (http://creativecommons.org/licenses/by$\mathrm{nc} / 4.0 /$ ) which permits unrestricted non-commercial use, distribution, and reproduction in any medium, provided the original work is properly cited.

Copyright (c) 2021 by The Korean Pediatric Society 
Table 1. Comparison of case reports of infants with multisystem inflammatory syndrome in children (MIS-C)

\begin{tabular}{|c|c|c|c|c|c|c|}
\hline \multirow{2}{*}{ Variable } & Infant 1 & Infant 2 & Infant 3 & Infant 4 & Infant 5 & Infant 6 \\
\hline & Kok et al. & Farias et al. ${ }^{3)}$ & Orlanski-Meyer et al. ${ }^{4)}$ & Acharyya et al. ${ }^{5)}$ & Raut et al. ${ }^{6)}$ & Jones et al. ${ }^{7)}$ \\
\hline \multicolumn{7}{|l|}{ Demographic } \\
\hline Country & Malaysia & Brazil & Israel & India & India & USA \\
\hline Age & 4 Months & 7 Months & 2 Months & 4 Months & 5 Months & 6 Months \\
\hline Sex & Female & Female & Female & Male & Male & Female \\
\hline \multicolumn{7}{|l|}{ Presenting features } \\
\hline Fever (duration) & $\sqrt{ }$ (3 Days) & $\sqrt{ }$ (3 Days) & $\sqrt{ }(10$ Days $)$ & $\sqrt{ }$ (4 Days) & $\sqrt{ }(5$ Days $)$ & $\sqrt{ }$ (4 Days) \\
\hline Rash & $\sqrt{ }$ & - & - & $\sqrt{ }$ & $\sqrt{ }$ & $\sqrt{ }$ \\
\hline Conjunctival injection & $\sqrt{ }$ & - & - & $\sqrt{ }$ & $\sqrt{ }$ & $\sqrt{ }$ \\
\hline Cracked/red lips & $\sqrt{ }$ & - & $\sqrt{ }$ & $\sqrt{ }$ & - & $\sqrt{ }$ \\
\hline Limb swelling & - & - & - & - & - & $\sqrt{ }$ \\
\hline Lymphadenopathy & - & - & - & $\sqrt{ }$ & - & - \\
\hline Respiratory & - & $\sqrt{ }$ & - & - & - & $\sqrt{ }$ \\
\hline Neurological ${ }^{\mathrm{b})}$ & - & $\sqrt{ }$ & - & - & - & - \\
\hline Gastrointestinal $^{\left({ }^{)}\right.}$ & $\sqrt{ }$ & $\sqrt{ }$ & $\sqrt{ }$ & - & - & - \\
\hline Cardiovascular $^{\mathrm{d})}$ & $\sqrt{ }$ & $\sqrt{ }$ & $\sqrt{ }$ & - & - & $\sqrt{ }$ \\
\hline Shock & $\sqrt{ }$ & $\sqrt{ }$ & $\sqrt{ }$ & - & - & - \\
\hline \multicolumn{7}{|l|}{ Investigations } \\
\hline RT-PCR SARS-CoV-2 & $\begin{array}{c}\text { Positive } \\
\text { (1st admission) }\end{array}$ & Positive & Negative & Positive & Positive & Positive \\
\hline SARS-CoV-2 Serology & Positive lgG & NA & Positive lgG & NA & NA & NA \\
\hline Total white cell count & Normal & $\uparrow$ & $\uparrow$ & Normal & Normal & NA \\
\hline Lymphocyte count & $\downarrow$ & $\downarrow$ & NA & NA & Normal & NA \\
\hline Platelet & $\downarrow$ & Normal & $\uparrow$ & $\downarrow$ & $\downarrow$ & Normal \\
\hline C-reactive protein & $\uparrow \uparrow$ & $\uparrow$ & $\uparrow \uparrow$ & $\uparrow \uparrow$ & $\uparrow \uparrow$ & $\uparrow \uparrow$ \\
\hline Ferritin & $\uparrow$ & $\uparrow$ & $\uparrow$ & NA & $\uparrow$ & NA \\
\hline Troponin I/B-NP & Normal & $\uparrow$ & $\uparrow$ & NA & $\uparrow$ & NA \\
\hline Albumin & $\downarrow$ & $\downarrow$ & $\downarrow$ & Normal & $\downarrow$ & $\downarrow$ \\
\hline Echocardiography & $\begin{array}{l}\text { Mild pericardial } \\
\text { effusion }\end{array}$ & $\begin{array}{l}\text { Mild pericardial effusion } \\
\text { with diffuse hypo- } \\
\text { contractility }\end{array}$ & Mild to moderate MR & $\begin{array}{l}\text { Diffuse ectasia of } \\
\text { coronary arteries }\end{array}$ & $\begin{array}{l}\text { Left main and LAD } \\
\text { coronary ectasia }\end{array}$ & Normal \\
\hline \multicolumn{7}{|l|}{ Therapeutic Intervention } \\
\hline Ventilation & $\sqrt{ }$ & $\sqrt{ }$ & - & - & - & - \\
\hline Inotropic support & $\sqrt{ }$ & $\sqrt{ }$ & - & - & - & - \\
\hline Aspirin & $\sqrt{ }$ & - & - & $\sqrt{ }$ & $\sqrt{ }$ & $\sqrt{ }$ \\
\hline Steroids & $\sqrt{ }$ & $\sqrt{ }$ & $\sqrt{ }$ & - & - & - \\
\hline IV immunoglobulin & $\sqrt{ }$ & - & $\sqrt{ }$ & $\sqrt{ }$ & $\sqrt{ }$ & $\sqrt{ }$ \\
\hline Interleukin antagonist & - & - & $\sqrt{ }$ & - & - & - \\
\hline Outcome & Alive & Died & Alive & Alive & Alive & Alive \\
\hline MIS-C criteria & Fulfilled & Fulfilled & Fulfilled & Fulfilled & Fulfilled & Fulfilled \\
\hline Kawasaki criteria & KDSS & - & KDSS & KD & Incomplete KD & KD \\
\hline
\end{tabular}

RT-PCR, reverse transcription-polymerase chain reaction; SARS-CoV-2, severe acute respiratory syndrome coronavirus-2; B-NP, B-type natriuretic peptide; IgG, immunoglobulin G; KD, Kawasaki disease; KDSS, Kawasaki disease shock syndrome; LAD, left anterior descending; MR, mitral regurgitation; IV, intravenous; NA, not applicable.

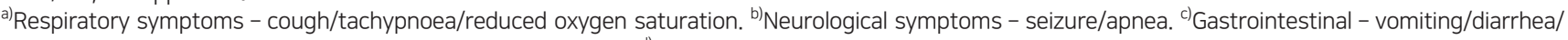
upper gastrointestinal bleeding/lower gastrointestinal bleeding. ${ }^{d)}$ Cardiovascular - features of pericarditis/myopericarditis/myocarditis. $\uparrow \uparrow C-$ reactive protein > $100 \mathrm{mg} / \mathrm{mL}$.

cases evidenced by positive antibody tests, and only a third had positive RT-PCR findings for SARS-CoV-2.9) A notable difference in the infants in this study was that a large proportion of infants (4 of $6,66 \%)$ had acute current SARS-CoV-2 infection as evidenced by positive RT-PCR tests rather than previous infections evidenced by positive antibody tests ( 2 of $6,33 \%$ ) (Table 1 ).
The younger age, female preponderance, and KD-like presentation in this cohort may suggest that these infants were genetically predisposed. SARS-CoV-2 may trigger activation of the inflammatory cascade in these infants. Another possible mechanism is the presence of autoantibodies due to T-cell recognition of selfantigens or viral antigens expressed on infected cells, resulting 
in a hyperimmune response state and cytokine storm caused by dysregulated immune responses. ${ }^{9)}$

KD usually responds well to IVIG therapy, and only 10\%$20 \%$ of cases are resistant to IVIG requiring either corticosteroid or biologics. On the other hand, the marked hyperinflammatory response in children with MIS-C often warrants combination therapy with both IVIG and corticosteroids.

This study had several limitations. First, we reviewed only a small number of infants and were not able to draw any definite conclusions regarding ethnicity or sex predilection. Moreover, comparing laboratory findings was difficult because some laboratory results were not available.

In conclusion, MIS-C shows similarities to KD, especially KDSS, which most likely is a consequence of dysregulated immune responses secondary to SARS-CoV-2 infection. Hence, the early recognition and diagnosis of MIS-C, followed by prompt treatment with IVIG and corticosteroids, will lead to favorable outcomes. The current diagnostic criteria for MIS-C do not adequately differentiate MIS-C from severe COVID-19; further refinement would better facilitate its diagnosis.

\section{Key message}

Question: Are multisystem inflammatory syndrome in children (MIS-C) and Kawasaki disease (KD) in infants, 2 sides of the same coin?

Finding: Here we report on a 4-month-old girl with MIS-C and signs of KD with shock. Most (83\%) infants with MIS-C had features of $\mathrm{KD}$, especially KD shock syndrome.

Meaning: MIS-C is similar to $\mathrm{KD}$, and likely is a consequence of dysregulated immune responses secondary to sudden acute respiratory syndrome coronavirus 2 infection.

Hing Cheong Kok, MD, Dinesh Nair, MBBS, Ke Juin Wong, MD, Siew Moy Fong, MBBS

Department of Paediatrics, Sabah Women and Children's Hospital, Ministry of Health Malaysia, Kota Kinabalu, Sabah, Malaysia

\section{Footnotes}

Conflicts of interest: No potential conflict of interest relevant to this article was reported.

ORCID:

Hing Cheong Kok (D) https://orcid.org/0000-0002-9781-5905

\section{References}

1. Centers for Disease Control and Prevention. Multisystem Inflammatory Syndrome in Children (MIS-C) Associated with Coronavirus Disease 2019 (COVID-19) [Internet]. Atlanta (GA): Centers for Disease Control and Prevention; [2020 Dec 25]. Available from: https://emergency.cdc. gov/han/2020/han00432.asp.

2. World Health Organisation. Multisystem inflammatory syndrome in children and adolescents temporally related to COVID-19 [Internet]. Geneva (Switzerland): World Health Organisation; 2020 May [2021 Jan 16]. Available from: https://www.who.int/news-room/commentaries/ detail/multisystem-inflammatory-syndrome-in-children-and-adolescentswith-covid-19.

3. Farias ECF, Justino MCA, Mello MLFMF. Multisystem inflammatory syndrome in a child associated with coronavirus disease in the Brazilian Amazon: fatal outcome in an infant. Rev Paul Pediatr 2020;38:e2020165.

4. Orlanski-Meyer E, Yogev D, Auerbach A, Megged O, Glikman D, Hashkes PJ, et al. Multisystem inflammatory syndrome in children associated with severe acute respiratory syndrome coronavirus- 2 in an 8-week-old infant. J Pediatric Infect Dis Soc 2020;9:781-4.

5. Acharyya BC, Acharyya S, Das D. Novel coronavirus mimicking Kawasaki disease in an infant. Indian Pediatr 2020;57:753-54.

6. Raut S, Roychowdhoury S, Bhakta S, Sarkar M, Nandi M. Incomplete Kawasaki disease as presentation of COVID-19 infection in an infant: a case report. J Trop Pediatr 2021;67:fmaa047.

7. Jones VG, Mills M, Suarez D, Hogan CA, Yeh D, Segal JB, et al. COVID-19 and Kawasaki disease: novel virus and novel case. Hosp Pediatr 2020;10: 537-40.

8. Whittaker E, Bamford A, Kenny J, Kaforou M, Jones CE, Shah P, et al. Clinical characteristics of 58 children with a pediatric inflammatory multisystem syndrome temporally associated with SARS-CoV-2. JAMA 2020;324:259-69.

9. Jiang L, Tang K, Levin M, Irfan O, Morris SK, Wilson K, et al. COVID-19 and multisystem inflammatory syndrome in children and adolescents. Lancet Infect Dis 2020;20:e276-88.

10. Holman RC, Belay ED, Christensen KY, Folkema AM, Steiner CA, Schonberger LB. Hospitalizations for Kawasaki syndrome among children in the United States, 1997-2007. Pediatr Infect Dis J 2010;29:483-8.

How to cite this article: Kok HC, MD, Nair D, Wong KJ, Fong SM. Multisystem inflammatory syndrome in children and Kawasaki disease in infants: 2 sides of the same coin? Clin Exp Pediatr 2021;64:599-601. https://doi.org/10.3345/cep.2021. 00913 